Musées, Patrimoine et Culture scientifiques et techniques

$145 \mid 2013$

janvier-février 2013

\title{
Anthropologie du mémoriel
}

\section{Anne-Claire Jolivet et Jérôme Lamy}

URL : http://journals.openedition.org/ocim/1177

DOI : 10.4000/ocim. 1177

ISSN : 2108-646X

\section{Éditeur}

OCIM

Édition imprimée

Date de publication : 1 janvier 2013

Pagination : 18-24

ISSN : 0994-1908

Référence électronique

Anne-Claire Jolivet et Jérôme Lamy, "Anthropologie du mémoriel », La Lettre de l'OCIM [En ligne],

145 | 2013, mis en ligne le 01 janvier 2015, consulté le 01 mai 2019. URL : http://

journals.openedition.org/ocim/1177; DOI : 10.4000/ocim.1177 


\section{Anthropologie du mémoriel}

\section{Anne-Claire Jolivet et Jérôme Lamy *}

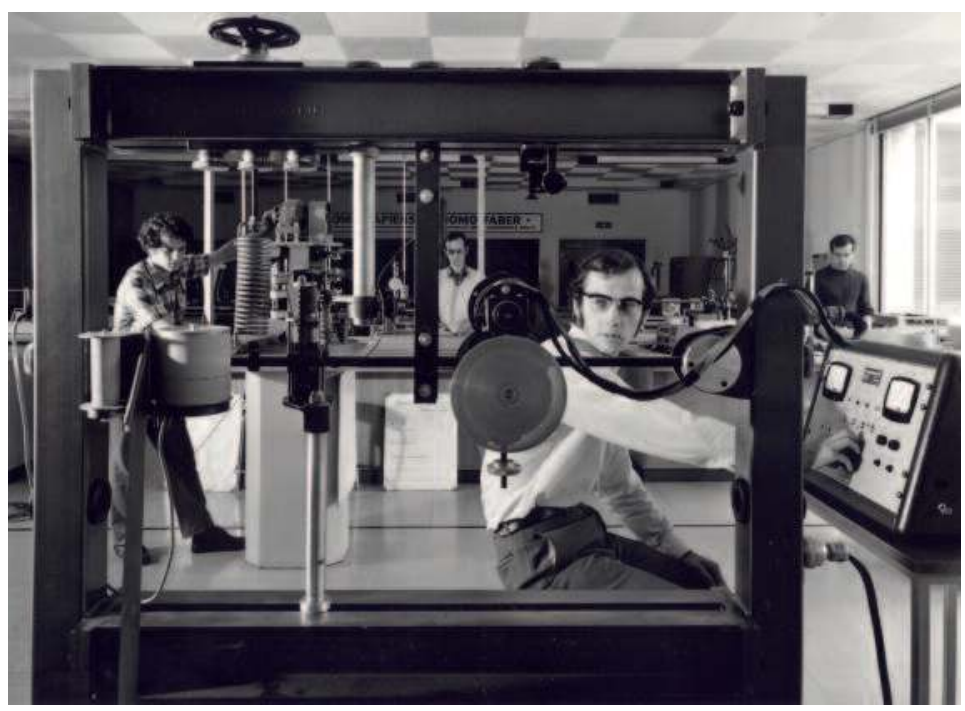

Élèves de l'École nationale supérieure de l'Aéronautique et de l'Espace (ENSAE) de Toulouse en 1972 () Jean Dieuzaide

* Anne-Claire Jolivet est responsable de la mission de sauvegarde du patrimoine scientifique et technique contemporain de Midi-Pyrénées

à l'université de Toulouse PRES, mission membre du réseau national PATSTEC anne-claire.jolivet@univ-toulouse.fr Jérôme Lamy est postdoctorant en histoire et anthropologie des sciences au laboratoire interdisciplinaire, Solidarités, Sociétés, Territoires (UMR 5193), centre d'Anthropologie sociale, université Toulouse II, EHESS jerome.lamy@laposte.net
Cette contribution présente les origines, la méthodologie et les premiers résultats du programme de recherche « Patrimoine immatériel du Toulouse scientifique moderne (1960-1990) » élaboré à l'intersection des préoccupations patrimoniales de la mission pour la sauvegarde du patrimoine scientifique et technique contemporain de Midi-Pyrénées et des questionnements autour de la mémoire des anthropologues de l'université de Toulouse.

\section{Jonctions institutionnelles : la mission PATSTEC de Midi-Pyrénées et les anthropologues}

Depuis 2004, la mission de sauvegarde du patrimoine scientifique et technique contemporain de Midi-Pyrénées, membre du réseau national PATSTEC (1) du musée des Arts et Métiers, récolte les témoins de l'activité de recherche du XXe siècle. Depuis janvier 2010, elle est intégrée au nouveau service de Diffusion des Sciences et des Techniques de l'université de Toulouse PRES. Outre la sauvegarde de ce patrimoine scientifique, ce service a pour mission de recenser et promouvoir les actions de culture scientifique réalisées par les établissements membres, de participer, à leur côté, aux rendez-vous culturels, de proposer un espace débat "Sciences et société », et d'ouvrir des modules de formation et des séminaires à la culture scientifique et technique. Cette structuration permet de concentrer la mission plus sereinement sur son cœur d'activité : l'inventaire des instruments et la récolte des témoignages des acteurs de la recherche. 
Ces pratiques patrimoniales concrètes permettent d'envisager une articulation solide à l'université et en particulier, à sa recherche en sciences humaines et sociales. La co-définition de sujets de recherche et de chantiers communs met en jeu des savoir-faire et des compétences muséologiques, anthropologiques, historiques et sociologiques. Le programme « Patrimoine immatériel du Toulouse scientifique moderne (19601990) » (PATOUS) s'est fondé sur un partenariat avec le Centre d'Anthropologie sociale du laboratoire interdisciplinaire Solidarités, Sociétés et Territoires (UMR 5193, université Toulouse II) et deux de ses chercheurs, Nicolas Adell (maître de conférences en Anthropologie) et Michel Grossetti (sociologue, directeur de recherche au CNRS). Le recrutement d'un post-doctorant (Jérôme Lamy) et de quatre vacataires (Lola Devolder, Ariela Epstein, Sébastien Plutniak et Josselin Tallec) a permis de définir le périmètre de l'enquête puis de la réaliser.

\section{Le patrimoine scientifique immatériel : essai de définition}

Le programme PATOUS propose, schématiquement, de nouer deux démarches. Il convient, d'une part, de coaguler les différentes actions patrimoniales à l'œuvre dans lesquelles le PRES est déjà largement impliqué. Il s'agit de retracer le parcours d'une trentaine de chercheurs. La constitution et l'analyse d'un corpus de témoignages de chercheurs ayant eu le cœur de leur activité professionnelle dans la même période (des années 1960 à 1990) est complémentaire de l'approche patrimoniale classique autour d'un sujet d'étude resserré (un instrument, un laboratoire, ou une vie). En se focalisant sur les récits de vie d'un certain nombre d'acteurs, il est possible de restituer les moments clés de l'histoire scientifique toulousaine, de repérer les scansions majeures, d'opérer une coupe transversale dans l'histoire des disciplines, et donc de placer finement des objets dans leur contexte socioéconomique.

D'autre part, PATOUS a pour ambition de mener une réflexion sur le processus menant à la « mise en patrimoine » des institutions scientifiques. Le patrimoine scientifique est, depuis le milieu des années 1980, un secteur particulièrement actif du champ mémoriel : d'abord centrés sur les grandes structures, les figures tutélaires de la science, les archives puis les instruments, les questionnements se sont peu à peu resserrés sur les pratiques quotidiennes de la recherche, les éléments diffus, les savoir-faire inscrits dans les corps et les connaissances tacites (2).

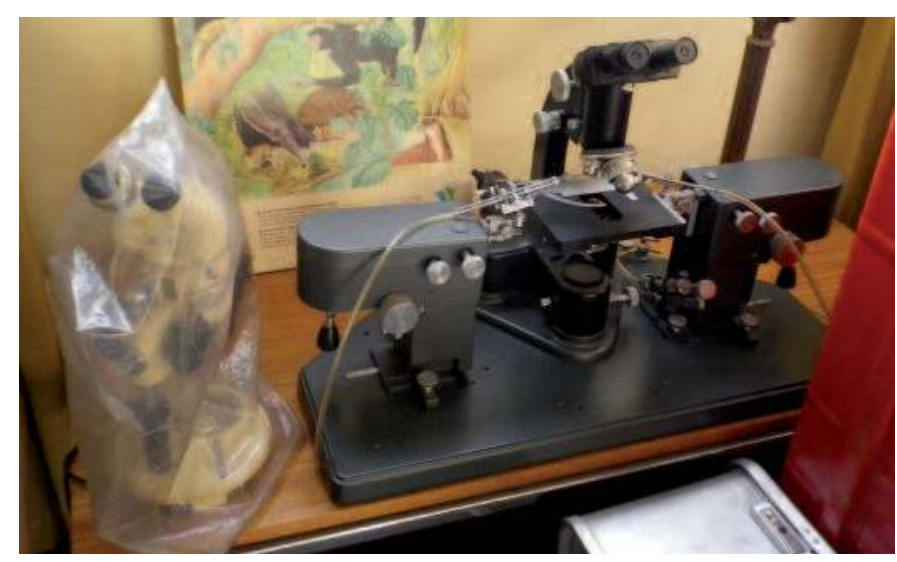

Micromanipulateur de Georges Larrouy (c) Mission sauvegarde du patrimoine scientifique et technique contemporain de Midi-Pyrénées

L'enjeu de cette reconfiguration des problématiques patrimoniales est double : d'une part rompre avec la démarche purement mémorielle, qui met en tension le travail de l'historien et réduit la science à une chronologie abruptement positive des découvertes ; d'autre part, définir une méthodologie spécifique pour saisir ce que les archives ignorent, tous ces gestes et ces savoirs implicites qui font l'activité scientifique.

La notion récente de patrimoine immatériel fournit de précieux éléments pour notre enquête : l'ensemble de ces "savoirs mouvants, ceux qui n'existent que dans les individus qui les mettent en ouvre à un moment donné » (3) ne peuvent être fixés par des objets ou des traces écrites ; ils ont en commun d'être transmis et recréés en permanence. L'UNESCO a adopté, en 2003, une convention valorisant ces savoirs immatériels et leurs réfractions dans le fonds commun de chaque culture. L'ensemble des connaissances ainsi visé est large : des pharmacopées traditionnelles aux théories cosmologiques, des modalités festives aux traditions culinaires (4), un nouveau « vertige des foisonnements » ${ }^{(5)}$ guette cet inventaire de l'infixable. Toutefois, l'intérêt heuristique de la notion permet d'envisager une déclinaison féconde pour le patrimoine scientifique. En interrogeant des chercheurs sur leur parcours, il est possible de faire émerger des éléments de pratiques et de discours mêlés qui donnent à voir ces savoirs immatériels, leur enchâssement dans la vie quotidienne (et non strictement scientifique) des personnes ainsi que la propre réflexivité de chacun d'eux par rapport à cette parole livrée. L'objectif a donc été fixé de recueillir des récits de vie des chercheurs toulousains, retraités ou encore en activité, ayant œuvré à l'université des années 1960 aux années 1990. 


\section{Méthodologie de l'enquête}

Le comité scientifique du projet PATOUS (composé d'acteurs patrimoniaux : Mission Archorales INRA, Service de l'Inventaire de la Région Midi-Pyrénées, Archives départementales et municipales, et de personnes reconnues par les acteurs nommés précédemment comme détenant un savoir « informel » sur l'histoire contemporaine de leur institution) avait rédigé une première liste d'acteurs prioritaires selon plusieurs critères :

- la reconnaissance de leur engagement et carrière scientifique, administrative et politique : des personnes identifiées par leurs pairs comme des «incontournables »; - leur sensibilité aux questions patrimoniales ;

- leur disponibilité potentielle ;

- la liste devait couvrir les disciplines clés de la recherche toulousaine contemporaine.

D’autres chercheurs étaient désignés comme des « ressources potentielles » susceptibles d'apporter des éléments de contextualisation. Finalement, cette frontière entre les deux catégories s'est brouillée. Ainsi le mathématicien Alain Rigal, prévu comme un témoin susceptible d'évoquer le cadre général des mathématiques à Toulouse, s'est mué en acteur-principal tant son témoignage s'est révélé informé et substantiel. Cette première liste des possibles interviewés balayait une gamme très large de disciplines : médecine, aéronautique/espace, informatique, mathématiques, archéologie, histoire, chimie, biologie, minéralogie, agronomie, météorologie, astronomie/astrophysique, physique, génie électrique, électronique, automatique, robotique, microscopie électronique, géographie, économie... Concrètement, nous n'avons pas couvert l'ensemble de ces champs disciplinaires : la médecine, la minéralogie, la météorologie ou la microscopie électronique n’ont pas encore été abordées en tant que telles. Certains interviewés n'ont pas souhaité répondre à nos sollicitations. D'autres, trop âgés, n'ont pu donner suite à nos demandes. Il nous a donc fallu recomposer une liste palimpseste en fonction des réponses qui nous sont arrivées. Cette recomposition du panel des interviewés a été menée à " périmètre constant » c'est-à-dire en tentant de préserver la cohérence d'ensemble (notamment en ce qui concerne la diversité disciplinaire) du projet.

Les entretiens se déroulent en deux temps. D'abord un entretien « libre » dans lequel le chercheur raconte, selon son propre rythme et sa propre perspective, l'histoire de son parcours. Cette reconstitution princeps a pour objectif de dégager un matériau mémoriel; elle distinguera les interviewés qui ont déjà mis en récit

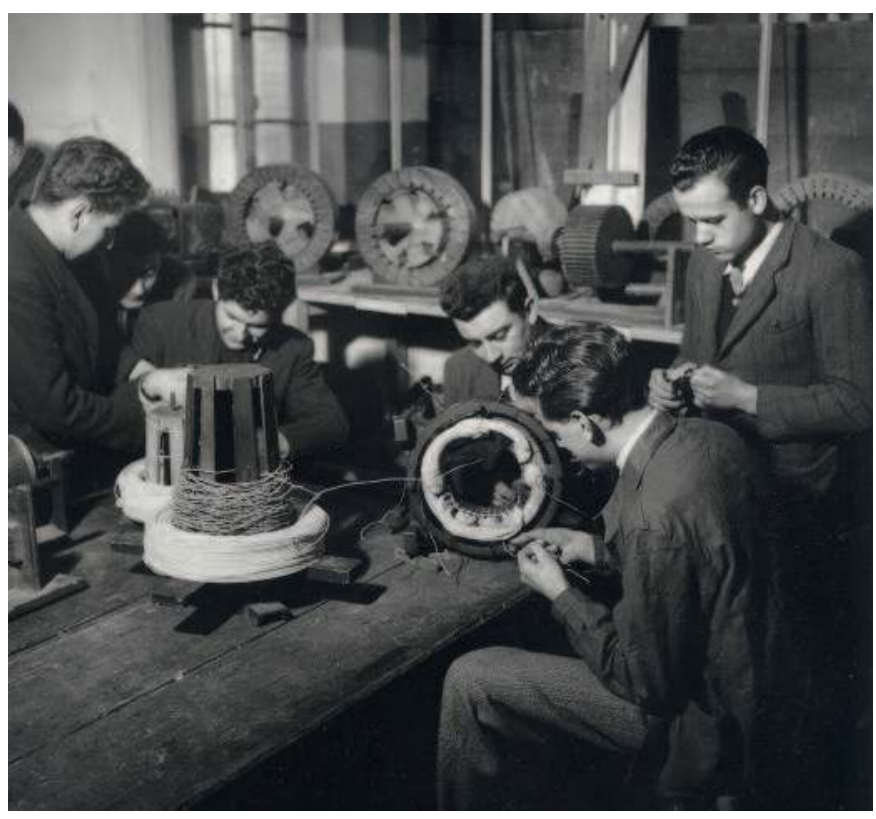

Étudiants en travaux pratiques d'électrotechnique à l'École nationale supérieure d'Électrotechnique et d'Hydraulique de Toulouse (aujourd'hui ENSEEIHT) en 1952

(c) Jean Dieuzaide

leur vie (articulations choisies, moments clés, initiations, généalogies intellectuelles...). Avant de mener les entretiens, nous avons collectivement discuté de la manière d'aborder les interviewés, de nouer les premiers contacts. Nous avons fourni un guide d'entretien, organisé en quatre grandes phases : cadre familial de l'enfance, formation (notamment le cursus universitaire), entrée en profession-carrière scientifique, bilan avec l'interviewé. Il s'agit d'un canevas assez lâche, destiné principalement à cadrer le récit de vie davantage quà l'infléchir. L'objectif est bien la production d'un récit de vie, dans ses cohérences et ses incohérences, ses strates mémorielles denses et ses zones allusives. Sur la trentaine d'entretiens prévus, une vingtaine a été réalisée.

Dans un deuxième temps nous avons réalisé un retour sur l'entretien avec des questions précises sur certains points pour comprendre jusqu'où la biographie a été (re)construite. Pour ces entretiens, il a été très important de laisser l'opportunité aux chercheurs de citer des noms qui ne sont pas apparus dans notre première liste et que nous avons documentés. Nous avons porté une grande attention aux motifs biographiques réitérés, ces topoï classiques (conversion, transformation, bifurcation) qui définissent une grammaire sommaire de l'autobiographie.

Outre cette constitution d'un corpus d'entretiens, il est possible de faire appel à des récits écrits spontanés qui viennent compléter des aspects moins connus et/ou 
moins immédiatement perceptibles des espaces sociaux questionnés. La collecte de documents (de toute nature : administratifs, scientifiques...) que les interviewés livrent spontanément ou bien que l'on sollicite complète les corpus d'entretiens.

Le programme PATOUS est centré sur une approche immatérielle du patrimoine scientifique et technique, mais il n'ignore pas non plus les aspects matériels de la mémoire savante. Lorsque dans l'enquête, nous sommes amenés à évoquer avec les interviewés des instruments avec lesquels ils ont travaillé nous en profitons pour faire un pré-inventaire de ces ressources artefactuelles (inventaire repris par la mission de sauvegarde du patrimoine scientifique et technique contemporain de Midi-Pyrénées). Bien sûr, les situations sont différentes selon les disciplines et ce questionnement sur les dispositifs instrumentaux n'a pas la même importance selon que nous évoquons le sujet avec un mathématicien ou avec un biologiste.

La phase de collecte des entretiens est aujourd'hui terminée. Nous abordons maintenant la constitution de grilles d'analyse pertinentes et la restitution des récits de vie. Nous avons fixé notre attention sur les cosmogonies personnelles et professionnelles des chercheurs qui tissent des relations complexes, des jeux d'échos multiformes entre la constitution d'un récitatif personnel sans cesse remanié (à l'image d'un palimpseste) et la pratique scientifique. Nous interrogeons notamment les dialectiques entre récit de vie et activités savantes. Plus globalement, nous inscrirons notre réflexion dans la problématique mémorielle qui occupe aujourd'hui une grande place dans les sciences humaines et sociales : la question du travail temporel (articulation des passés, des présents et des futurs), les disjonctions ou les concordances des rythmes personnels et professionnels ainsi que les investissements contrastés dans la création des récits biographiques (de la quasi-professionnalisation de l'autoportrait du scientifique au rejet le plus complet de cette forme d'expression).

\section{Premiers résultats : parcours de mathématiciens}

Afin de rendre plus sensible le travail analytique en cours, nous présentons brièvement une partie de l'enquête : les entretiens avec les mathématiciens des différents campus toulousains (Henri Caussinus, statisticien à l'université Paul Sabatier, Alain Rigal, qui travaille sur les mathématiques appliquées, Pierre Ettinger qui s'est notamment consacré aux probabilités, Maryvonne Spiesser et Michel Guillemot qui ont œuvré pour l'histoire des mathématiques à l'université
Paul Sabatier et enfin, Philippe Carbonne qui s'est occupé de l'enseignement des mathématiques à l'université Toulouse Le Mirail). Nous avions, au débat, une liste de chercheurs à interviewer en priorité. Leurs parcours sont très différents, les champs de recherche dans lesquels ils sont intervenus sont également très variés. Il est néanmoins possible de repérer deux grands axes qui organisent les récits de vie de ces mathématiciens toulousains. D'une part, leurs trajectoires permettent de rendre compte de l'évolution des structures universitaires : les grandes thématiques de recherche, les transformations de l'enseignement, la diffusion, dans les trois universités toulousaines, des recherches mathématiques forment une armature institutionnelle particulièrement précise. D'autre part, il est possible d'exploiter les récits de vie des mathématiciens en restituant la façon dont ils relatent leur carrière, leur goût pour les mathématiques, leurs pratiques d'enseignant, leur conception du métier, toutes ces indications qui donnent à voir la pratique mathématique à l'université de Toulouse dans sa complexité et sa diversité.

L'histoire des institutions universitaires toulousaines dédiées aux mathématiques depuis la fin des années 1950 jusquà l'orée des années 2000 constitue la trame la plus dense des entretiens ${ }^{(6)}$. Les récits des mathématiciens interviewés sont convergents sur un point essentiel : au lendemain de la guerre, la recherche en mathématiques à Toulouse est faible pour ne pas dire inexistante. Les mathématiques pures, en particulier, semblent ne pas rencontrer d'écho particulier. À partir des années 1960, trois pôles s'organisent.

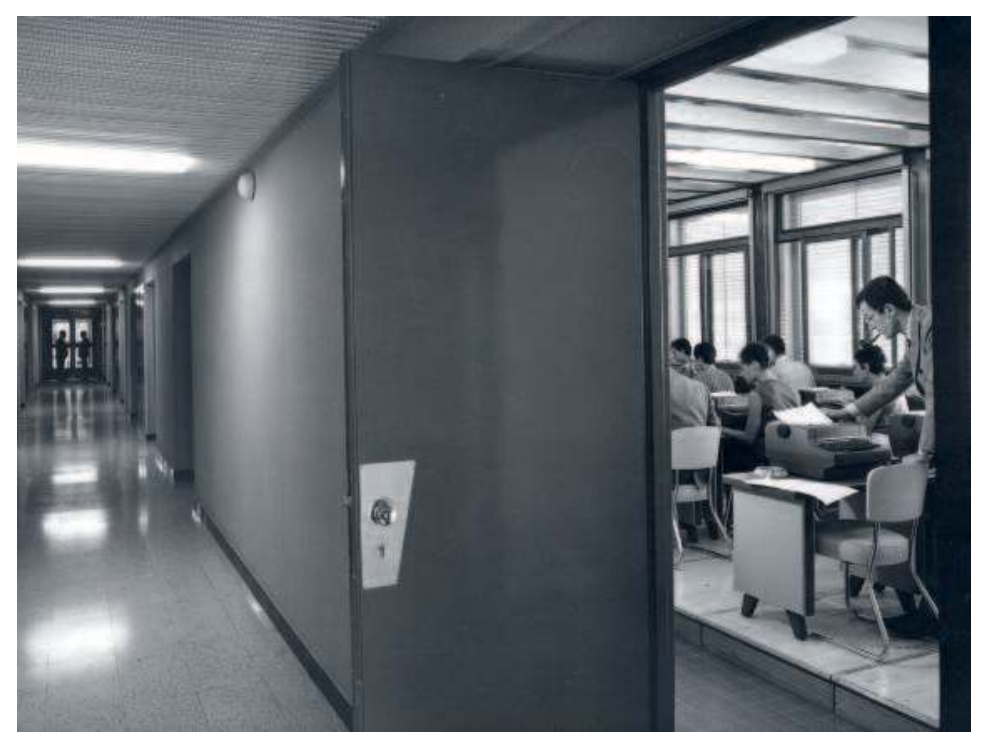

Institut de calcul numérique (Instcalculnum) de la faculté des Sciences de Toulouse (aujourd'hui UPS) en 1966 () Jean Dieuzaide 
Autour du physicien Émile Durand, le calcul numérique devient très vite un secteur de pointe ${ }^{(7)}$. La faculté des Sciences de Toulouse se dote ainsi dès 1957 d'un IBM 650. C'est autour de cette nouvelle technologie qu'Émile Durand crée l'Institut de Calcul numérique, épicentre des mathématiques appliquées à Toulouse. Il est important de noter que le calcul numérique se développe à Rangueil sous l'impulsion d'un physicien, qui oriente ses travaux vers les mathématiques : on mesure donc ici l'importance des échanges interdisciplinaires. Surtout Émile Durand est entouré d'une vingtaine de personnes dont certaines (comme les dessinateurs ou les programmeurs) sont essentiellement affectées à l'élaboration de ses livres.

Un deuxième pôle est également solidement organisé, il s'agit des statistiques et probabilités. Là encore, comme pour le calcul numérique, l'ancrage est ancien. Dans le sillage du professeur Deltheil (qui enseignait les probabilités à Toulouse avant la Seconde Guerre mondiale), Roger Huron, médecin et mathématicien, organise dans les années 1950 le laboratoire de Statistique de l'université de Toulouse. En contact avec les médecins, les biologistes, ses travaux sont très éclectiques.

Le troisième pôle, qui n'est qu'émergeant dans les années 1950, c’est l'informatique. Mené par Michel Laudet, qui est un élève de Émile Durand, ce secteur connaît une forte croissance. Michel Laudet, dans une perspective très stratégique, vise d'abord une promotion institutionnelle de la discipline : il fait ainsi venir des personnalités comme Jacques-Louis Lyons qui donne des cours de DEA dans les années 1960. L’une des spécificités du laboratoire de Laudet réside dans la construction en interne d'ordinateurs.

Voici, donc, pour Rangueil et à grands traits, l'architecture des institutions mathématiques à Toulouse à la veille des évènements de mai 1968. Une nouvelle fois, les récits des mathématiciens interrogés lors de l'enquête sont convergents pour décrire la complète réorganisation des structures après le printemps de manifestations et de grèves : l'université s'est massifiée, l'afflux d'étudiants est considérable, le recrutement des enseignants s'accélère, les responsables des laboratoires sont renouvelés. Les lignes entre les différentes branches des mathématiques ne sont plus aussi nettes qu'avant. D'abord, les mathématiques pures font leur apparition (timidement), les statistiques montent franchement en puissance tout au long des années 1970. Roger Huron, comme la plupart de ceux qui occupaient les fonctions dirigeantes auparavant, quitte la direction de son laboratoire.

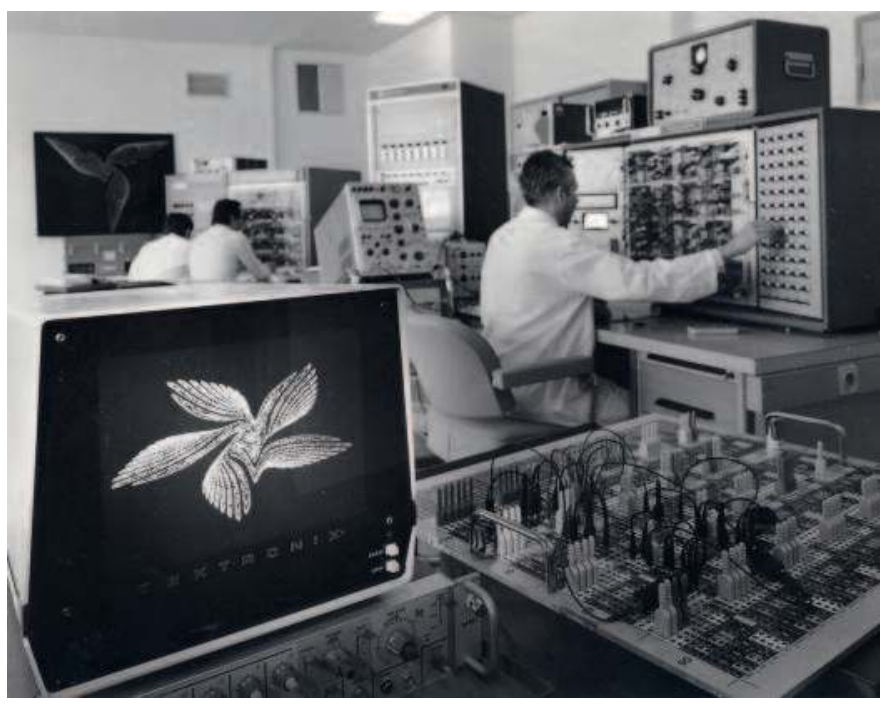

"Ensemble du dispositif associé au calculateur analogique et résultat graphique sur l'écran de la simulation numérique d'une zone stochastique dans une récurrence presque conservative", au laboratoire d'Automatique et de ses Applications Spatiales (aujourd'hui LAAS) en 1973.

( ) Jean Dieuzaide

C'est Henri Caussinus, alors jeune maître de conférences, qui se charge de réorganiser le travail d'équipe. Voici ce qu'il dit : "À ce moment-là, on a décidé de créer un laboratoire en bonne et due forme, avec un conseil de laboratoire et des statuts. Nous étions le premier laboratoire à avoir un statut » (8).

Les précédents laboratoires étaient de pure forme, il ne s'agissait pas de mener des recherches en commun ; après 1968, les travaux sont coordonnés (au mieux) et l'on peut véritablement parler d'équipe. Les changements sont plus nombreux encore dans le domaine du calcul numérique. L'ancienne structure édifiée par Émile Durand est absorbée par une nouvelle équipe dirigée par Marc Attéia et devient, en 1977, le laboratoire d'Analyse numérique. Dès lors et à partir des années 1980, l'organisation des mathématiques à l'université Paul Sabatier est assez classique avec un laboratoire d'analyse numérique, un laboratoire de statistiques et de probabilités, un laboratoire de mathématiques pures et enfin, l'informatique qui s'est autonomisée, tant du point de vue des recherches que de l'enseignement.

Lultime phase de transformation est la création dans les années 1990 de l'Institut de Mathématiques de Toulouse. Voulu par Henri Caussinus, cette structure se calque sur les «Bell Laboratories » des ÉtatsUnis, et associe pour les mathématiques, « une recherche fondamentale de très haut niveau, une recherche finalisée et des applications industrielles » $(9)$. 
Telles sont donc, en quelques mots, les transformations, vécues et perçues par les acteurs eux-mêmes, des mathématiques toulousaines sur le campus de Rangueil des années 1970 aux années 1990. Les logiques de fragmentation de la discipline, de polarisation des recherches (notamment mathématiques appliquées/mathématiques fondamentales) ont toujours joué un rôle dans l'équilibre de la recherche toulousaine, mais on remarquera que la tendance lourde, esquissée dès le début des années 1990, est à un rassemblement des équipes, par-delà les orientations disciplinaires. Il ne faut pas oublier dans ce paysage mathématique toulousain le cas du Mirail. Lorsque l'université s'est scindée en trois, après 1968, les découpages disciplinaires (faculté de Droit, faculté de Lettres, faculté des Sciences) ont été très largement reconduits. Toutefois, une filière "Mathématiques et Sciences Humaines » est créée au Mirail. Elle regroupe des linguistes, des mathématiciens, des informaticiens. Cette structure connaît des reconfigurations nombreuses, et se rapproche des Sciences économiques et de la Gestion.

Les entretiens avec les mathématiciens toulousains ont donc permis de mettre en exergue l'organisation de la discipline sur les différents campus. On remarquera d'abord que les spécialités (notamment la statistique) sont historiquement ancrées. Il existe des traditions de recherche, des filières spécifiques qui malgré les transformations diverses, subsistent sous une forme ou sous une autre. On notera ensuite que les mathématiques se sont peu à peu organisées en véritables laboratoires : les travaux d'équipe, les coordinations des chercheurs sont peu à peu devenus le quotidien de la recherche mathématique (assez loin de l'image stéréotypée du scientifique isolé).

\section{Premier bilan}

Ainsi, l'enquête anthropologique et historique menée auprès des mathématiciens toulousains a permis de saisir - et c'est là une "denrée » rare pour les chercheurs en sciences sociales - des moments de l'histoire universitaire qui sont à la fois singuliers et généraux. Singuliers parce qu'ils appartiennent à l'histoire personnelle des mathématiciens interrogés : ils sont chargés de leurs émotions, de leurs affects, ils sont pris dans des réseaux interpersonnels complexes (des amitiés, des ruptures, des conflits). Généraux parce qu'ils se rattachent aux mouvements de l'institution universitaire : les pesanteurs de l'avant 1968, les transformations des années 1970, l'éclatement des structures (les dénominations des facultés, UER, UFR... n'ont cessé de changer).

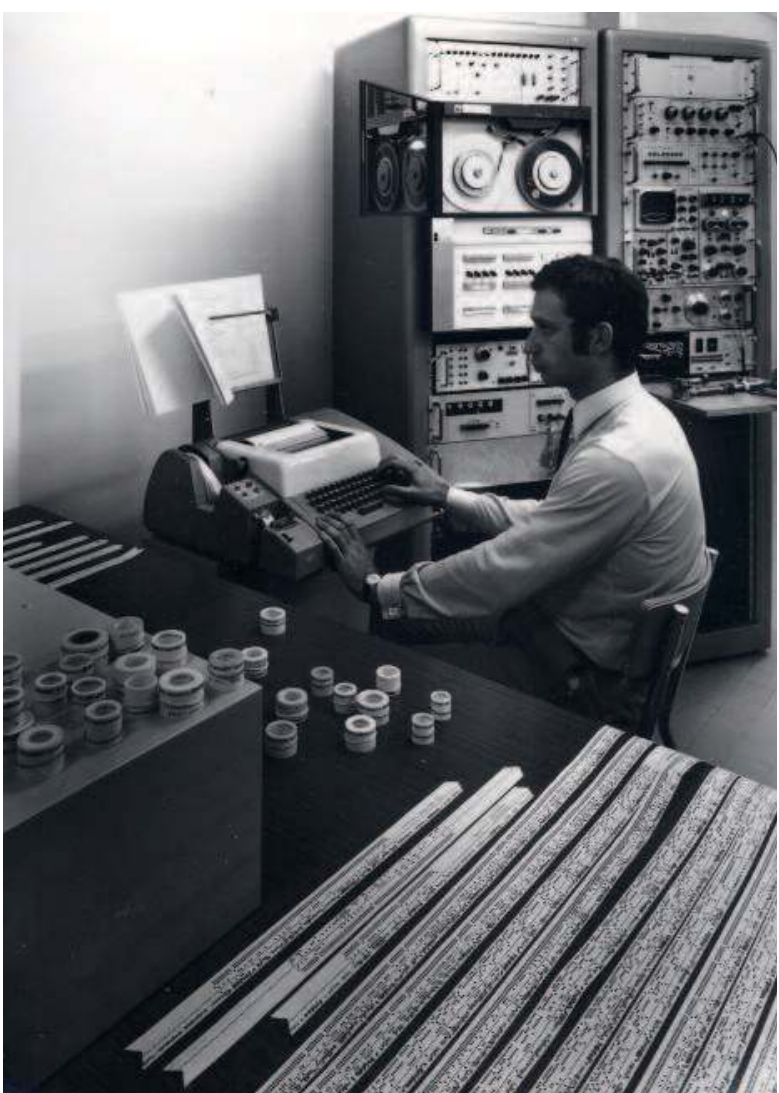

Programmation d'une série d'ordres sur un téléimprimeur à la faculté des sciences de Toulouse (aujourd'hui UPS) en 1969

(C) Jean Dieuzaide

Le récit des mathématiciens est donc tressé de ces fils personnels et historiques, de ces moments quasiment indicibles (le bonheur exprimé par Henri Caussinus à l'évocation des heures d'enseignement) et de ces mouvements collectifs (les souvenirs de Pierre Ettinger sur la ronéotype fonctionnant à plein en mai 1968). C'est ce maillage complexe, du particulier et du général, que nous analysons maintenant pour mieux comprendre comment se construit la mémoire. Que retenons-nous d'une vie ? De quels éléments se sert-on pour construire un récit? Ces questions, qui sont au cœur des sciences sociales, n'appellent pas d'interprétations univoques, nous devons accepter de toujours les poser (avec le moins d'a priori possible) et de se laisser surprendre par la réponse.

\section{Notes}

(1) Ballé, C., Cuenca, C. et Thoulouze, D. Patrimoine scientifique et technique, un projet contemporain. Paris : La Documentation Française, 2010 . 
(2) Pour une synthèse sur les tensions entre histoire et mémoire : Lamy, J. Tension histoire/mémoire dans la valorisation du patrimoine scientifique et technique : une perspective critique, Cahiers François Viète, série II, n³, 2010, pp. 7-35.

(3) Adell, N. Anthropologie des savoirs. Paris : Armand Colin, 2011, p. 188.

(4) www.unesco.org/culture/ich/index.php?lg=fr\&pg=00022

(5) L'expression est d'Alphonse Dupront (Histoire de la psychologie collective et vie du temps, in L'Encyclopédie Française, tome XX, 1959, p. 6), reprise par Alain Corbin dans un article : Le vertige des foisonnements. Esquisse panoramique d'une histoire sans nom, Revue d'histoire moderne et contemporaine, tome 39-1, 1992, pp. 103-126). Corbin théorisait ainsi sa propre définition d'une histoire des sensibilités qu'il a développée en travaillant sur des objets aussi divers que l'odorat, le « désir du rivage » et les sonorités des cloches (Corbin, A. Le miasme et la jonquille. L'odorat et l'imaginaire social aux XVIII et XIX ${ }^{e}$ siècles. Paris : Aubier, 1982 ; Corbin, A. Le territoire du vide. L'Occident et le désir du rivage (1750-1840). Paris : Flammarion, 1990 ; Corbin, A. Les cloches de la terre. Paysage sonore et culture sensible dans les campagnes au XIXe siècle. Paris : Flammarion, 2000).
(6) Tous les entretiens menés avec les mathématiciens ont contribué à reconstituer l'histoire des mathématiques au sein de l'université de Toulouse. Le témoignage d'Alain Rigal a été particulièrement précieux, en présentant une vue ample des structurations successives de l'activité mathématique (entretien avec Alain Rigal, 3 mars 2011).

(7) Dans son maître-ouvrage sur l'informatique en France de la Seconde Guerre mondiale au Plan Calcul, Pierre-Éric Mounier-Kuhn retrace les grandes lignes du parcours d'Émile Durand : Mounier-Kuhn, P.-É. L'informatique en France de la Seconde Guerre mondiale au Plan Calcul. L'émergence d'une science. Paris : Presses de l'université Paris-Sorbonne, 2010, pp. 262-263

(8) Entretien avec Henri Caussinus, 16 février 2011.

(9) Archives personnelles d'Henri Caussinus, Projet d'Institut de Mathématiques de Toulouse par le groupe de prospectives pour les mathématiques, 21 mai 1991. 